\title{
High-dose OxyContin to treat pain associated with bone metastasis in patients with small-cell lung cancer: a case study report
}

This article was published in the following Dove Press journal:

Drug Design, Development and Therapy

19 January 2016

Number of times this article has been viewed

\author{
Tao Zhou',* \\ Xia Zhang ${ }^{2, *}$ \\ Yan Dong' \\ Feifei Zhuang' \\ Fengquan Jiang ${ }^{3}$ \\ Jinming $\mathrm{Yu}^{4}$ \\ Bin Zhang ${ }^{1,4}$
}

'Department of Oncology, The First Affiliated Hospital of Dalian Medical University, Dalian, ${ }^{2}$ Department of Oncology, Shandong Cancer Hospital and Institute, Shandong University, Jinan, ${ }^{3}$ Laboratory of Medicine, The First Affiliated Hospital of Dalian Medical University, Dalian, Liaoning, ${ }^{4}$ Department of Radiation Oncology, Shandong Cancer Hospital and Institute, Shandong University, Jinan, Shandong, People's Republic of China

*These authors contributed equally to this work

\begin{abstract}
Pain management is an important topic that has received extensive attention from clinical practitioners. Nearly all patients with malignant tumors suffer pain at the advanced stage of their disease. Oxycodone is a first-line choice for treating moderate-tosevere cancer-related pain, and OxyContin, a controlled-release oxycodone hydrochloride tablet, is internationally recognized as a safe and effective opioid analgesic. OxyContin has the characteristics of both immediate release and sustained release, with a time to onset and peak similar to those of immediate-release morphine. It acts on both $\mu$ and $\kappa$ receptors and has been shown to be effective in treating different types of pain, especially neuropathic pain, theoretically without a dose cap. However, the dose is limited in clinical applications due to various factors that are likely to affect its analgesic effect and reduce patient quality of life. Cooperation with a patient's family members is required during the treatment of cancer pain. Chronic cancer pain has a long disease course, which could easily cause complex psychological symptoms due to their important role in the pain experience. Pain is controllable, and patients have a right to not experience pain. An optimal living state can be achieved through collaboration between physicians and patients. Rational personalized treatment of cancer pain can improve patient quality of life, relieve pain, and help prolong patient survival. This article reports the treatment procedure and adverse reactions in a patient who was treated with high-dose OxyContin, with the aim of providing a reference for other clinical practitioners.
\end{abstract}

Keywords: pain, OxyContin, bone metastasis, palliative care

\section{Introduction}

Pain has been listed as the fifth vital sign. Nearly all patients with malignant tumors suffer pain at the advanced stage of their disease. The treatment of cancer pain affects patient quality of life, which is closely related to cancer therapy. According to the principles of the three-step analgesic ladder, oxycodone is a first-line choice for treating moderate-to-severe cancer pain, and its controlled-release formulation, OxyContin, is a key drug for treating moderate-to-severe pain. ${ }^{1}$ Oxycodone has no capping dose due to the absence of a "ceiling" effect. However, the oxycodone dosage is restricted for various reasons in clinical practice. This article reports the use of high-dose OxyContin to treat a patient who was admitted to our hospital, with the aim of providing a reference for the clinical application of oxycodone. The time of the first OxyContin dose was used as the baseline in this report. This study was approved by the First Affiliated Hospital of Dalian Medical University, and written informed consent was obtained from the patient. 


\section{Patient information}

A 57-year-old male patient was hospitalized for chemotherapy on November 20, 2013, at 1 year and 11 months after being diagnosed with small-cell lung cancer in the left lung. The patient underwent fiber optic bronchoscopy at the First Affiliated Hospital of Dalian Medical University on December 9, 2011. Pathology results indicated small-cell lung cancer, and a diagnosis of small-cell lung cancer of the left lung was made (limitation period). After the diagnosis was confirmed, the patient underwent an etoposide chemotherapy regimen (VP-16 plus cisplatin). The efficacy evaluation after 2 weeks indicated stable disease (SD). The patient refused radiotherapy. After an additional cycle of chemotherapy, the patient voluntarily terminated treatment due to poor compliance. The chest computed tomography (CT) examination on July 3, 2012, indicated a space-occupying mass at the hilus of the left lung, which was accompanied by obstructive pneumonia in the upper lobe of the left lung and nodules in the left lung. Compared with the CT results from February 14, 2012, mediastinal lymph node metastases and an enlarged lesion were observed, indicating disease progression. With the patient's consent, VP-16 capsules were administered as oral chemotherapy, and SD was achieved after two treatment cycles. The treatment was stopped again due to poor patient compliance. In late July 2012, the patient suffered hoarseness and was hospitalized because of chest tightness and shortness of breath. The imaging examination suggested disease progression. The patient began irinotecan and carboplatin chemotherapy on July 28, 2012, and completed four treatment cycles. The lung CT examination on April 3, 2013 indicated a space-occupying mass at the hilus of the left lung, accompanied by obstructive pneumonia in the upper lobe of the left lung. Compared with the CT results from January 6 , 2013, the space-occupying mass at the hilus of the left lung was enlarged, and the inflammation in the upper lobe of the left lung was aggravated; furthermore, both nodules in the left lung were considered malignant based on increased size, whereas the mediastinal lymph node metastases remained stable. The efficacy evaluation indicated progressive disease (PD). The patient began the third-line chemotherapy regimen CAV (ifosfamide + epirubicine + vincristine) on April 7, 2013, and completed four treatment cycles. In October 2013, the patient experienced headache and pain in the right shoulder. Chest and abdominal CT examinations and head magnetic resonance imaging indicated tumor progression. Bone electroconvulsive therapy and cervical magnetic resonance imaging examinations revealed no clear bone metastasis. After consultation among different departments in our hospital, the patient began one cycle of the fourth-line pemetrexed combined cisplatin chemotherapy regimen (pemetrexed in combination with lobaplatin) on October 21, 2013.

\section{Previous analgesic treatment}

The patient was given oral ibuprofen sustained-release capsules at a dosage of $600 \mathrm{mg}$ every 12 hours for right shoulder pain (numeric rating scale $[\mathrm{NRS}]=3$ ), and the pain was relieved (NRS =1).

At the time of admission, the pain manifested as dull and pricking sensations in the neck, shoulder, chest, and back, with mainly pricking pain (NRS $=6$ ). The pain was mostly evoked by activity and was slightly relieved upon rest, with 2-4 episodes of breakthrough pain (NRS =7) everyday. The patient exhibited emotional irritability due to the pain, which affected his diet, sleep, and daily activities and led to his unwillingness to communicate with others.

The patient received an assessment and opioid titration immediately after hospitalization. According to the recommendations in the National Comprehensive Cancer Network guidelines for treating cancer pain, the incremental dose was calculated based on the total dose of opioid drugs within the first 24 hours, and the dose was increased for a specific time or need.

\section{Use of OxyContin}

The patient was first administered OxyContin on November 20, 2013, with titration starting at $10 \mathrm{mg}$ Q12h. The analgesic dosage was determined the next day at $40 \mathrm{mg}$ Q12h and was increased to $120 \mathrm{mg}$ Q12h after 1 month. The dosage was increased to $660 \mathrm{mg}$ Q12h in January 2014, but there was no improvement in pain management associated with the dosage increase. Instead, the patient began to have acid reflux after taking the drug. Therefore, the dosage was reduced to $600 \mathrm{mg}$ Q12h. Later, because the analgesic effect did not last for 12 hours and the patient refused to take the drug at an incremental dose each time, $600 \mathrm{mg}$ Q8h was attempted. Acceptable pain management was achieved through dosage adjustment and combination with adjuvant medication. The patient was given $50 \mathrm{mg}$ oral morphine immediate-release tablets, $2-5$ times per day, at the onset of breakthrough pain. The NRS score was 2-3 after analgesic treatment, with only difficulty in urination during treatment. However, at the end stage of the disease, the patient was in poor physical condition, with a performance status (PS) score of 3-4 and brain metastasis. It was unclear whether there was spinal cord metastasis that caused spinal cord compression, and there was insufficient evidence to prove whether the symptoms were related to the drugs. No other obvious adverse reactions were observed. During the period when 
Table I Dose adjustment of OxyContin and use of adjuvant medication during disease progression

\begin{tabular}{|c|c|c|c|c|c|c|}
\hline Date & $\begin{array}{l}\text { NRS } \\
\text { before } \\
\text { adjustment }\end{array}$ & $\begin{array}{l}\text { Dosage after } \\
\text { adjustment } \\
\text { (total daily dose) }\end{array}$ & $\begin{array}{l}\text { NRS after } \\
\text { adjustment }\end{array}$ & $\begin{array}{l}\text { Number of onsets } \\
\text { of breakthrough } \\
\text { pain and treatment } \\
\text { (times a day) }\end{array}$ & Adjuvant medication & $\begin{array}{l}\text { Treatment for adverse } \\
\text { reactions }\end{array}$ \\
\hline $\begin{array}{l}\text { November } 21 \\
2013\end{array}$ & 7 & 80 & 3 & Morphine $20 \mathrm{mg} \times 2$ & Ibuprofen + carbamazepine & $\begin{array}{l}\text { No significant constipation, } \\
\text { nausea, or vomiting; } \\
\text { no delirium or other } \\
\text { opioid toxicity observed }\end{array}$ \\
\hline $\begin{array}{l}\text { December } 2 \text {, } \\
2013\end{array}$ & 7 & 160 & 2 & Morphine $20 \mathrm{mg} \times 5$ & Ibuprofen + gabapentin & Same as above \\
\hline $\begin{array}{l}\text { December 27, } \\
2013\end{array}$ & 7 & 240 & 2 & Morphine $20 \mathrm{mg} \times(4-5)$ & Ibuprofen + gabapentin & Same as above \\
\hline $\begin{array}{l}\text { January } 23, \\
2014\end{array}$ & 7 & 600 & 2 & Morphine $20 \mathrm{mg} \times(4-6)$ & Ibuprofen + gabapentin & Same as above \\
\hline $\begin{array}{l}\text { February } 4, \\
2014\end{array}$ & 8 & $\mathrm{I}, 320$ to $\mathrm{I}, 200$ & 1 & Morphine $50 \mathrm{mg} \times(2-5)$ & Alprazolam & Urinary retention? \\
\hline $\begin{array}{l}\text { February I4, } \\
2014\end{array}$ & 8 & 1,800 & 1 & Morphine $50 \mathrm{mg} \times(\mathrm{I}-3)$ & Alprazolam & Urinary retention? \\
\hline
\end{tabular}

Note: "?" refers to being unsure whether the urinary retention was caused by morphine.

Abbreviation: NRS, numeric rating scale.

OxyContin was used, ibuprofen controlled-release capsules, carbamazepine, gabapentin, and alprazolam were utilized as adjuvant medications based on symptoms and clinical reactions (Table 1).

\section{Follow-up treatment}

After two cycles of chemotherapy with pemetrexed combined with lobaplatin, the efficacy assessment indicated PD. After considering the report on chemotherapy-sensitive genes and the toxicity prediction for the patient, he began to take oral Gimeracil and Oteracil potassium capsules as fifth-line monotherapy on December 2, 2013. The efficacy evaluation after two treatment cycles indicated PD. Multiple bone metastases were observed. The patient received one cycle of Docetaxel combined with Cisplatin as sixth-line chemotherapy. The patient was hospitalized on February 8, 2014, with a PS score of 3 and poor appetite, and received best supportive care. The patient was pronounced clinically dead on February 20, 2014. The cause of death was end-stage lung cancer and respiratory failure (Table 2).

\section{Discussion}

Oral administration is the dosing route preferred by the World Health Organization and the European Association for Palliative Care for drugs that treat cancer pain. OxyContin is a controlled-release oxycodone hydrochloride tablet for oral administration. It acts on both $\mu$ and $\kappa$ receptors and has been shown to be effective in treating different types of pain (eg, somatic pain, visceral pain, and neuropathic pain), especially neuropathic pain. The drug has characteristics of both immediate release and sustained release, with a time to onset and peak similar to those of immediate-release morphine. OxyContin is currently the first-choice drug for treating moderate-to-severe pain. ${ }^{2}$

Comprehensive and dynamic assessments of cancer pain form the basis of personalized dosing regimens. ${ }^{3}$ The initial assessment is usually completed within 24 hours after a patient is hospitalized. A comprehensive assessment is conducted again after 3 days of analgesic treatment or when the cancer pain has been stably relieved. In principle, the pain assessment is performed at least twice each month. The patient in this report received a complete evaluation after being hospitalized, and dosage titrations, pain assessments, and adverse event monitoring were performed during the entire cancer pain treatment process. During this period, the patient and his family cooperated, and the patient showed good compliance, unlike in the initial treatment stage. At the end stage of the disease, the analgesic effect of OxyContin at $600 \mathrm{mg}$ Q12h did not last for 12 hours, and the patient refused to take additional dose increments at each drug administration. Therefore, oral administration of $600 \mathrm{mg}$ OxyContin Q8h was attempted, and the daily dose reached $1,800 \mathrm{mg}$. This dosage was sufficient for daily pain management, but breakthrough pain episodes could not be avoided.

The treatment of drug-related adverse reactions requires attention during pain management. The most common adverse reactions of opioid drugs are gastrointestinal reactions such as nausea, vomiting, and constipation. ${ }^{4}$ In the clinic, when metoclopramide poorly controls vomiting, a low-dose oral hormone can be added to increase patient 


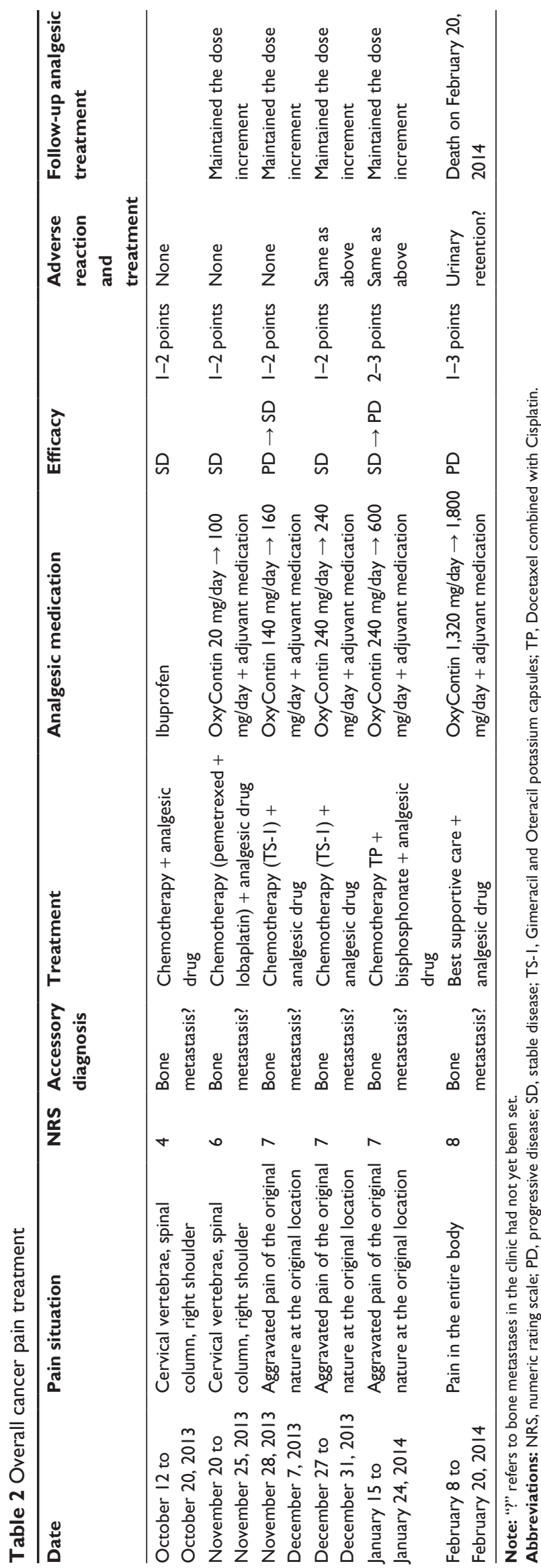

appetite and alleviate adverse reactions. The stool softener polyethylene glycol is the first-choice drug for preventing constipation. It has mild adverse reactions and is well tolerated, thereby reasonably reducing the use of stimulant laxatives. Respiratory depression is a life-threatening side effect of opioid drugs, especially when they are used at high doses, when a combination of analgesic drugs is used, or when high-dose morphine is intrathecally administered. Despite the low incidence of respiratory depression, this side effect should not be ignored. Sensitive monitoring methods are currently unavailable. A clinically feasible method is to monitor oxygen saturation in blood, but this method has low sensitivity. The development of a simple and easy $\mathrm{CO}_{2}$ monitoring technique is needed. The patient described herein did not show evident gastrointestinal reactions during treatment. When the daily dose of OxyContin reached 1,800 mg, which is equivalent to $2,400 \mathrm{mg}$ of morphine, the patient did not exhibit respiratory depression or other mental symptoms that are usually caused by morphine, such as drowsiness, euphoria, and cognitive changes. ${ }^{5}$ This observation suggests that the safety of OxyContin is more clinically acceptable. The patient suffered difficulty in urination at the end stage of the disease. He had brain metastases; therefore, it is unknown whether the difficulty in urination was caused by spinal cord compression by spinal cord metastases. There was no evidence suggesting that this symptom was related to the administered medications. Drug administration every 8 hours was an empirical attempt at pain management. Although this dosage is not recommended in the guidelines, it is necessary to ascertain the general condition of each patient in clinical practice, and a personalized analysis and approach can be adopted in the application of analgesic drugs; the primary goal is to ensure an acceptable quality of life for the patient and to enable the patient to live with dignity. ${ }^{6}$ During the treatment of cancer pain, chemotherapy was constantly attempted in an effort to control tumor progression and thereby alleviate patient suffering. During the episodes of $\mathrm{SD}$, the cancer pain was under control, indicating that chemotherapy is an important treatment for cancer pain. Although data from this patient indicate that high-dose OxyContin is safe, we are unsure whether this dose can be utilized in other patients; even at a lower dose, this drug may cause serious, even life-threatening, adverse reactions in other patients. Based on communications at national conferences, cases in which high doses were utilized have been reported by other organizations, but recognized evidence is not yet available. Moreover, we cannot determine the highest clinical dose of OxyContin based on a few cases because we cannot blindly 
pursue the goal of completely eradicating pain while ignoring potential concerns regarding side effects, which may have terrible consequences. Certainly, we expect more data, particularly from large-scale clinical research, to support our conclusion. There is much to be learned about the treatment of cancer pain, and we will continue to explore this aspect of patient management.

Pain management is an important topic that has received extensive attention from clinical practitioners. Cooperation with a patient's family members is required during cancer pain treatment. Chronic cancer pain has a long disease course, which could easily cause complex psychological symptoms due to their important role in the pain experience. Psychological factors and emotional states can affect the sensation of pain. Psychological drug interventions include tricyclic antidepressants, benzodiazepine sedatives, and other nonmedication-based therapies. ${ }^{7}$ Adjuvant drugs were added during the treatment of this patient based on his situation, but they failed to achieve the expected desirable effect. The intended analgesic effect was achieved by adjusting the OxyContin dose. Regarding nondrug-based treatments, the patient should be guided in self-adjustment, with an emphasis on educating the patient and their family members to strengthen self-administered pain assessments and management by teaching them how to assess the intensity and nature of their pain and to monitor adverse reactions. To effectively prevent pain, patient compliance with the treatment regimen should be improved, their confidence in the analgesic treatment should be enhanced, and they should be kept in a pleasant mood. ${ }^{8}$

In summary, pain is controllable, and patients have a right to experience no pain. An optimal living state can be achieved through collaboration between physicians and patients. Rational personalized treatment of cancer pain can improve patient quality of life, relieve pain, and help prolong patient survival.

\section{Acknowledgments}

This work was supported by the Shandong Provincial Natural Science Foundation (Project No ZR2013HL048), the Natural Science Foundation of Shandong Academy of Medical Sciences (Project No 2013-35), and the Science Foundation of The First Affiliated Hospital of Dalian Medical University (2014QN004).

\section{Disclosure}

The authors report no conflicts of interest in this work.

\section{References}

1. Hesselbarth S, Hermanns K, Oepen P. Prolonged-release oxycodone/ naloxone in opioid-naïve patients - subgroup analysis of a prospective observational study. Expert Opin Pharmacother. 2015;16:457-464.

2. Hayashi T, Ikehata S, Matsuzaki H, et al. Influence of serum albumin levels during opioid rotation from morphine or oxycodone to fentanyl for cancer pain. Biol Pharm Bull. 2014;37:1860-1865.

3. Xiao Y, Liu J, Huang XE, et al. Clinical study on fluvoxamine combined with oxycodone prolonged-release tablets in treating patients with moderate to severe cancer pain. Asian Pac J Cancer Prev. 2014;15: 10445-10449.

4. Poelaert J, Koopmans-Klein G, Dioh A, et al. Treatment with prolongedrelease oxycodone/naloxone improves pain relief and opioid-induced constipation compared with prolonged-release oxycodone in patients with chronic severe pain and laxative-refractory constipation. Clin Ther. 2015; 37:784-792.

5. Zhang WZ, Yu WJ, Zhao XL, He BX. Pharmacoeconomics evaluation of morphine, MS contin and oxycodone in the treatment of cancer pain. Asian Pac J Cancer Prev. 2014;15:8797-8800.

6. Lee JJ, Lee MK, Kim JE, et al. Pain relief scale is more highly correlated with numerical rating scale than with visual analogue scale in chronic pain patients. Pain Physician. 2015;18:E195-E200.

7. Porcerelli JH, Bornstein RF, Porcerelli D, Arterbery VE. The complex role of personality in cancer treatment: impact of dependency-detachment on health status, distress, and physician-patient relationship. J Nerv Ment Dis. 2015;203:264-268.

8. Cajanus K, Kaunisto MA, Tallgren M, Jokela R, Kalso E. How much oxycodone is needed for adequate analgesia after breast cancer surgery: effect of the OPRM1 118A>G polymorphism. J Pain. 2014;15:1248-1256.

\section{Publish your work in this journal}

Drug Design, Development and Therapy is an international, peerreviewed open-access journal that spans the spectrum of drug design and development through to clinical applications. Clinical outcomes, patient safety, and programs for the development and effective, safe, and sustained use of medicines are a feature of the journal, which

\section{Dovepress}

has also been accepted for indexing on PubMed Central. The manuscript management system is completely online and includes a very quick and fair peer-review system, which is all easy to use. Visit http://www.dovepress.com/testimonials.php to read real quotes from published authors.

Submit your manuscript here: http://www.dovepress.com/drug-design-development-and-therapy-journal 